\title{
Ein Leben für das Auge
}

\section{Adrian Ritter}

Freier Journalist

Balder Gloor hat die Augenheilkunde in der Schweiz als Forscher und Klinikdirektor mitgeprägt. Auch nach seiner Emeritierung bleibt er der Ophthalmologie verbunden. In einem kürzlich erschienenen Buch zeichnet er die Tätigkeit seines Grossvaters Arthur Gloor nach, der 1899 bis 1954 in Solothurn als Augenarzt wirkte.

Die Medizin verschwindet nicht aus dem Blickfeld von Balder Gloor. Seit der 85-Jährige und seine Frau vom Haus am Zürichberg in eine Wohnung umgezogen sind, leben sie im selben Gebäude wie die Direktion des UniversitätsSpitals Zürich ihre Büros hat. Von ihrer Wohnung aus blicken Balder und Marie-Louise Gloor auf das Careum-Bildungszentrum im Nebengebäude. Die dortige medizinische Bibliothek war Balder Gloor in den vergangenen Jahren oft nützlich. Seit seiner Emeritierung als Professor für Ophthalmologie und Direktor der Klinik für Augenheilkunde am UniversitätsSpital Zürich Ende 1999 hatte Gloor mehr Zeit, sich seinem Interesse für die Geschichte der Medizin und der Augenheilkunde zu widmen. In rund vierjähriger Arbeit hat er den einzigartigen Nachlass des Solothurner Augenarztes Arthur Gloor aufbereitet und in einer soeben erschienenen Publikation zugänglich gemacht (vgl. Kasten). Arthur Gloor war der Grossvater von Balder Gloor.

Blicken wir zurück: Arthur Gloor verschreibt seinem damals 12-jährigen Enkel nicht nur die erste Brille, son-

Am Kantonsspital St. Gallen begegnet er seiner zukünftigen Frau Marie-Louise Schindler, die dort ebenfalls als Assistenzärztin tätig ist.

dern hinterlässt ihm bei seinem Tod 1954 auch seine ophthalmologischen Lehrbücher. Balder Gloor ist da-

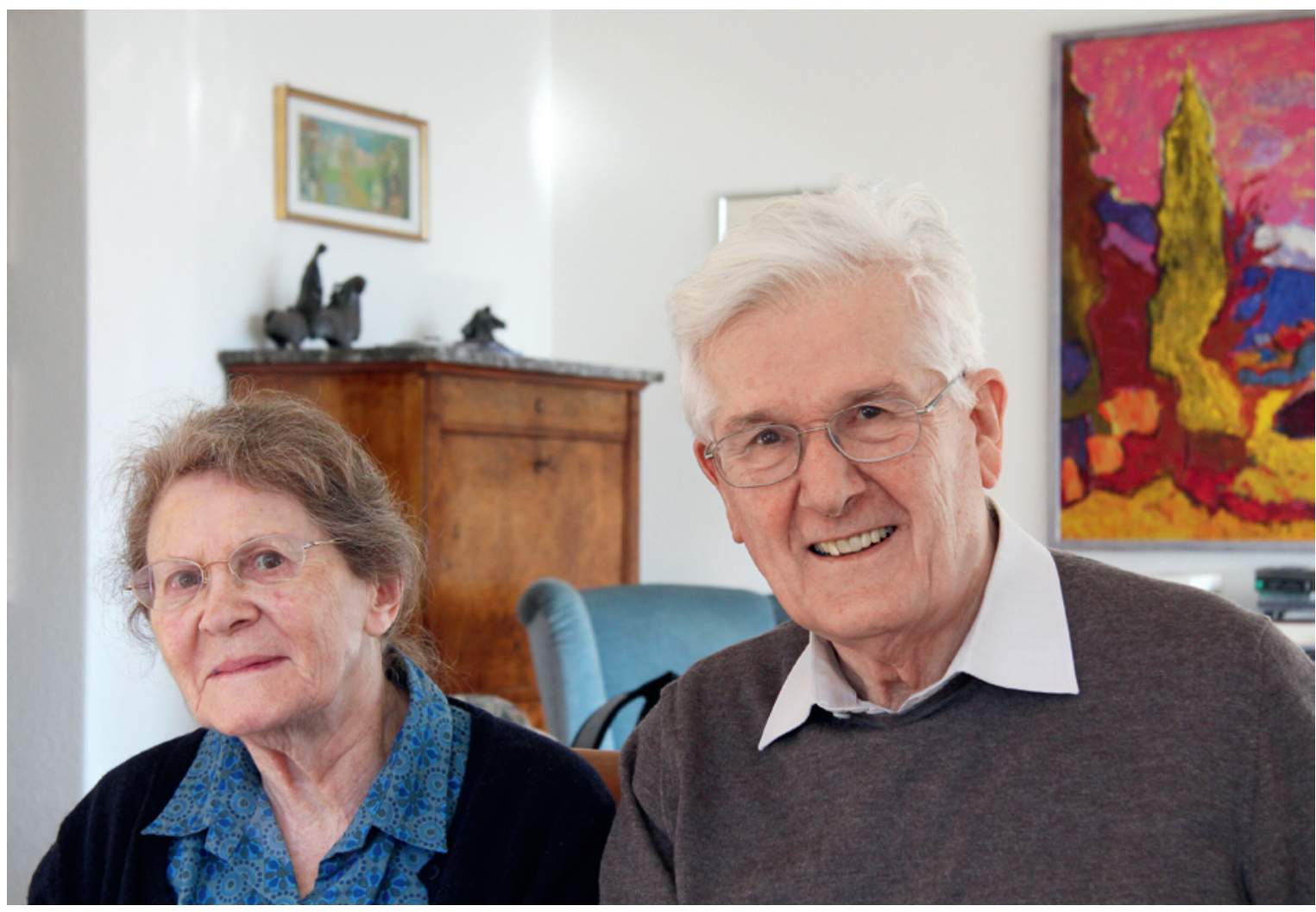

Balder Gloor und seine Frau Marie-Louise. 
mals im zweiten Jahr seines Medizinstudiums an der Universität Bern. Er ist breit interessiert, zweifelt noch an der Studienwahl und schwänzt schon mal eine medizinische Vorlesung, um eine Veranstaltung in Geschichte oder Philosophie zu besuchen. Die klinischen Praktika überzeugen ihn dann aber, das richtige Studienfach gewählt zu haben. Die beste Vorlesung und

\section{Balder Gloor ist einer der Ersten, die Stamm- zelltransplantationen nach Verätzungen der Hornhaut durchführen.}

den besten Dozenten - Hans Goldmann - findet er in der Augenheilkunde, in die er sich später vertieft. Zuerst aber will er sich als Assistenzarzt solide Kenntnisse in Pathologie und Innerer Medizin aneignen. Am Kantonsspital St. Gallen begegnet er seiner zukünftigen Frau Marie-Louise Schindler, die dort ebenfalls als Assistenzärztin tätig ist. Sie wird ihn später auch in seiner ophthalmologischen Forschung unterstützen.

\section{Nachhaltige Fellowships}

Balder Gloor verschreibt sich nach der Assistenzzeit ganz der Augenheilkunde, unter anderem während eines Aufenthalts an der Washington University School of Medicine. Er wird Abteilungsleiter an der Universitäts-Augenklinik Bern und ab 1974 Professor und Vorsteher der Universitäts-Augenklinik Basel. Dort führt er 1974 das schweizweit erste durchorganisierte Curriculum für die Facharzt-Weiterbildung ein. Von Basel wechselt er 1985 nach Zürich und wird Direktor der dortigen Universitäts-Augenklinik. Bis zu seiner Emeritierung und darüber hinaus engagiert er sich in verschiedenen Ämtern, etwa als Herausgeber von Fachzeitschriften sowie als Präsident der Schweizerischen Ophthalmologischen Gesellschaft.

Auch in der Forschung macht sich Balder Gloor einen Namen. Er kann unter anderem nachweisen, welche Zellen und welche Mechanismen nach einer Netzhautoperation für die gelegentlich auftretende pathologische Zellproliferation auf der Netzhaut und im Glaskörper verantwortlich sind. Beim Glaukom zeigt er, dass früheste Symptome nicht bei der Einschränkung des Gesichtsfeldes zu suchen sind - «das ist bereits der Anfang vom Ende»-, sondern bei Veränderungen am Sehnerv. Zudem ist Balder Gloor einer der Ersten, die Stammzelltransplantationen nach Verätzungen der Hornhaut durchführen. Dabei vergisst Gloor nie, dass die Segnungen der modernen Medizin nicht allen Menschen gleichermassen zur Verfügung stehen. Nach der Emeritierung lanciert und realisiert er im International Council of Ophthal- mology (ICO) eine Idee, die zu einem grossen Erfolg wird: die ICO Fellowships. Rund 1000 junge Augenärztinnen und Augenärzte aus weniger entwickelten Ländern haben seit 2001 davon profitiert und einen Aufenthalt an einer Augenklinik im Ausland absolvieren können. Mit ihrem neuen Wissen kehren die Fellows anschliessend in ihre Heimat zurück. «Die ICO-Fellowships sind das Nachhaltigste, was ich in meinem Leben geschaffen habe. Sie haben mitgeholfen, die Augenheilkunde in zahlreichen Ländern deutlich zu verbessern", sagt Balder Gloor.

\section{Zeit für die Geschichte}

Nach dem Aufbau des Fellowship-Programms widmet sich Balder Gloor ab 2007 vor allem seinem Interesse an historischen Fragen und wertet den Nachlass seines Grossvaters aus. Der Fundus, den die Familie der Zentralbibliothek Solothurn übergeben hat, ist wohl einmalig: Die Arbeitsjournale mit Krankengeschichten und Zeichnungen umfassen rund 45000 Patientinnen und Patienten, die Arthur Gloor von 1899 bis 1954 in seiner eigenen Praxis und am Bürgerspital Solothurn behandelte. «Das Buch soll keine Glorifizierung der Arbeit des Grossvaters sein», sagt Balder Gloor. «Es ging mir nicht um die Frage, ob er ein guter oder schlechter $\mathrm{Au}$ genarzt war. Ich wollte diesen wertvollen Fundus nicht vergessen gehen lassen und aufzeigen, wie sich die damalige Augenheilkunde von der heutigen unterscheidet.»

Der Katalog der rund 240 Erkrankungen und Verletzungen des Auges habe sich in den rund 100 Jahren kaum verändert, stellt Gloor fest, die Diagnosestellung allerdings sehr wohl: Ophthalmoskop und Spaltlampe seien zwar immer noch wichtige Werkzeuge, mit Computertomographie und MRI liessen sich heute

Die Risiken von Augenoperationen werden weiter sinken und bessere Medikamente zur Behandlung des erhöhten Augendrucks entwickelt werden.

aber sogar einzelne Schichten der Netzhaut darstellen. Dabei lauern gemäss Gloor aber auch Gefahren: Vor lauter technischer Hilfsmittel bei der Diagnose sei in den vergangenen Jahrzehnten die Schulung des Beobachtungsvermögens zunehmend vernachlässigt worden.

Dass sein Grossvater die Augen und Krankheiten seiner Patienten so akribisch zeichnerisch festhielt, war nicht nur ein freudvolles Ausleben eines Talentes, ist Gloor überzeugt: «Das Zeichnen schärfte auch die Beobachtungsgabe - damals der Schlüssel zur Dia- 
Augenheilkunde von 1899 bis 1954 - gelebt und gezeichnet

Der Augenarzt Arthur Gloor hat in seiner eigenen Praxis und als Belegarzt am Bürgerspital Solothurn von 1899 bis 1954 rund 45000 Patientinnen und Patienten behandelt. Deren Leiden wie auch Lebensumstände hat der Arzt in Wort und Bild akribisch festgehalten.

In mehrjähriger Arbeit hat Balder Gloor die Aufzeichnungen seines Grossvaters gesichtet und ausgewertet - bis hin zu Statistiken der behandelten Krankheiten und Verletzungen. Der Hauptteil des Buches widmet sich den einzelnen Augenerkrankungen und den damaligen Möglichkeiten der Behandlung. Darin verwoben finden sich die Notizen von Arthur Gloor zu den Lebensumständen seiner Patientinnen und Patienten.

In weiteren Buchkapiteln bettet der Autor die Berichte seines Grossvaters in den Kontext der Geschichte der Augenheilkunde ein - damals die erste Spezialisierung innerhalb der Medizin. Der sorgfältig gemachte, ästhetische Text- und Bildband erlaubt es, in die Geschichte der Medizin wie auch in die Lebenswelt der Menschen der Region Solothurn in der ersten Hälfte des 20. Jahrhunderts einzutauchen. Ein wertvolles Stück Zeitgeschichte.

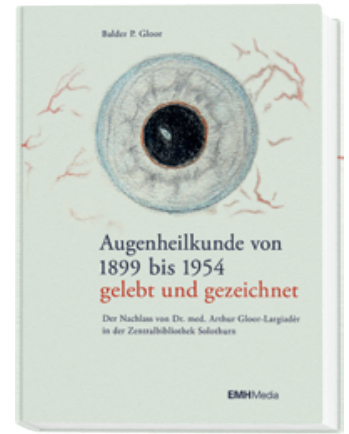

Balder P. Gloor

Augenheilkunde von 1899 bis 1954 gelebt und gezeichnet Der Nachlass von Dr. med. Arthur Gloor-Largiadèr in der Zentralbibliothek Solothurn

Basel: EMH Schweizerischer Ärzteverlag AG; 2017. 495 Seiten, $58 \mathrm{CHF}$.

ISBN 978-3-03754-097-8 gnose.» Der Enkel hat das ärztliche Zeichnen denn auch bis ans Ende seiner ärztlichen Tätigkeit selber gepflegt.

\section{Zufriedene Patienten}

Was die Behandlungsmöglichkeiten in der Ophthalmologie anbelangt, so ergab sich ein grosser Fortschritt nach dem Zweiten Weltkrieg, als sich entzündliche Augenkrankheiten dank Antibiotika plötzlich viel erfolgreicher behandeln liessen. Hinzu kamen gemäss Balder Gloor weitere neue wirksame Substanzen wie Hormone, aber auch künstliche Linsen und vor allem enorme Fortschritte in den Operationstechniken - etwa in der Netzhautchirurgie. Viele Augenerkrankungen seien heute gut behandelbar oder gar heilbar. «Die Ophthalmologie ist eine medizinische Disziplin mit vielen zufriedenen, dankbaren Patientinnen und Patienten", blickt Gloor auf seine Tätigkeit zurück.

Wie wird sich die Augenheilkunde wohl in Zukunft entwickeln? Balder Gloor glaubt nicht, dass Entwicklungen wie die Digitalisierung und die Personalisierte Me-

adrianritter[at]gmx.ch dizin die Augenheilkunde grundlegend verändern werden. Weitere Fortschritte seien aber durchaus absehbar: Die Gentherapie werden helfen, Augen-Erbkrankheiten zu verhindern und zu heilen. Die Risiken von Augenoperationen werden weiter sinken und bessere Medikamente zur Behandlung des erhöhten Augendrucks entwickelt werden. Dass Staroperationen dank neuer Medikamente bald überflüssig werden, sei allerdings nicht zu erwarten: «Das hat man schon mehrmals prophezeit, ohne dass es eingetreten ist», so Balder Gloor. Mit den rasanten Entwicklungen in der Robotik sieht er die Entwicklung eines künstlichen Sehapparates bald von der Utopie zur Realität werden. Um ein solch «künstliches Auge» - welches ganz anders aussehen könnte als ein natürliches Auge - auch mit dem Gehirn verbinden zu können, stehe allerdings noch viel Arbeit bevor.

Mehr als der Blick in die Zukunft fasziniert Balder Gloor heute aber der Blick in die Vergangenheit. Mit der $\mathrm{Pu}-$ blikation Augenheilkunde von 1899 bis 1954 - gelebt und gezeichnet über das Wirken seines Grossvaters hat er selber einen wertvollen Einblick in die Geschichte der Augenheilkunde geliefert. 